Vietnam Journal of Mechanics, VAST, Vol.42, No. 1 (2020), pp. $29-42$

DOI: https://doi.org/10.15625/0866-7136/14628

\title{
THEORETICAL AND EXPERIMENTAL ANALYSIS OF THE EXACT RECEPTANCE FUNCTION OF A CLAMPED-CLAMPED BEAM WITH CONCENTRATED MASSES
}

\author{
Nguyen Viet Khoa ${ }^{1, *}$, Dao Thi Bich Thao ${ }^{1}$ \\ ${ }^{1}$ Institute of Mechanics, VAST, Hanoi, Vietnam \\ E-mail: nvkhoa@imech.vast.vn
}

Received: 13 November 2019 / Published online: 01 March 2020

\begin{abstract}
This paper establishes the exact receptance function of a clamped-clamped beam carrying concentrated masses. The derivation of exact receptance and the numerical simulations are provided. The proposed receptance function can be used as a convenient tool for predicting the dynamic response at arbitrary point of the beam acted by a harmonic force applied at arbitrary point. The influence of the concentrated masses on the receptance is investigated. The numerical simulations show that peak in the receptance will decrease when there is a mass located close to that peak position. The numerical results have been compared to the experimental results to justify the theory.
\end{abstract}

Keywords: receptance, frequency response function, concentrated mass.

\section{INTRODUCTION}

The receptance function is very important in vibration problems such as control design, system identification or damage detection since it interrelates the harmonic excitation and the response of a structure in the frequency domain. The receptance method was first introduced by Bishop and Johnson [1]. This method has been developed and applied widely in mechanical systems and structural dynamics. Milne [2] proposed a general solution of the receptance function of uniform beams which can be applied for all combinations of beam end conditions. Yang [3] derived the exact receptances of nonproportionally damped dynamic systems. In this work an iteration procedure is developed based on a decomposition of the damping matrix, which does not require matrix inversion and eliminate the error caused by the undamped model data. Lin and Lim [4] proposed the receptance sensitivity with respect to mass modification and stiffness modification from the limited vibration test data. Mottershead [5] investigate the measured zeros from frequency response functions and its application to model assessment and updating. Gurgoze [6] presented the receptance matrices of viscously damped systems

(C) 2020 Vietnam Academy of Science and Technology 
subject to several constraint equations. In this paper, the frequency response matrix of the unconstrained system and the coefficient vectors of the constraint equations was used to obtain the frequency response matrix of the constrained system. Gürgöze and Erol [7] established the frequency response function of a damped cantilever simply supported beam carrying a tip mass. In this paper, the frequency response function was derived by using a formula established for the receptance matrix of discrete linear systems subjected to linear constraint equations, in which the simple support was considered as a linear constraint imposed on generalized co-ordinates. Burlon et al. [8] derived an exact frequency response function of axially loaded beams with viscoelastic dampers. The method relies on the theory of generalized functions to handle the discontinuities of the response variables, within a standard 1D formulation of the equation of motion. In another work, Burlon et al. [9] presented an exact frequency response of two-node coupled bending-torsional beam element with attachments. Karakas and Gürgöze [10] extended the work in [3] in which the receptance matrix was obtained directly without using the iterations as presented in [3] to form the receptance matrix of non-proportionally damped dynamic systems. Muscolino and Santoro [11] developed the explicit frequency response functions of discretized structures with uncertain parameters. Recently, the authors of this paper [12] presented the exact formula of the receptance function of a cracked beam and its application for crack detection. However, the exact form of frequency response function of a beam with concentrated masses has not been established yet.

The aim of the present paper is to present an exact receptance function of a clampedclamped beam carrying an arbitrary number of concentrated masses. The proposed formula of receptance function is simple and can be applied easily to investigate the dynamic response of beam at an arbitrary point under a harmonic force applied at any point along the beam. The influence of concentrated masses on the receptance of the clamped-clamped beam is investigated. The comparison between numerical simulations and experimental results have been carried out to justify the proposed method.

\section{THEORETICAL BACKGROUND}

Considering the Euler-Bernoulli beam carrying concentrated masses subjected to a force as shown in Fig. 1, the governing bending motion equation of the beam can be extended from [13] as follows

$$
E I y^{\prime \prime \prime \prime}+\left[m+\sum_{k=1}^{n} m_{k} \delta\left(x-x_{k}\right)\right] \ddot{y}=\delta\left(x-x_{f}\right) f(t),
$$

where $E$ is the Young's modulus, $I$ is the moment of inertia of the cross sectional area of the beam, $\mu$ is the mass density per unit length, $m_{k}$ is the $k^{\text {th }}$ concentrated mass located at $x_{k}, y(x, t)$ is the bending deflection of the beam at location $x$ and time $t, f(t)$ is the force acting at position $x_{f}, \delta\left(x-x_{f}\right)$ is the Dirac delta function. Symbols " ' " and "' " denote differentials with respect to $x$ and $t$, respectively.

Eq. (1) can be rewritten in the form

$$
E I y^{\prime \prime \prime \prime}+m \ddot{y}=\delta\left(x-x_{f}\right) f(t)-\sum_{k=1}^{n} m_{k} \delta\left(x-x_{k}\right) \ddot{y} .
$$


Theoretical and experimental analysis of the exact receptance function of a clamped-clamped beam with concentrated masses 31

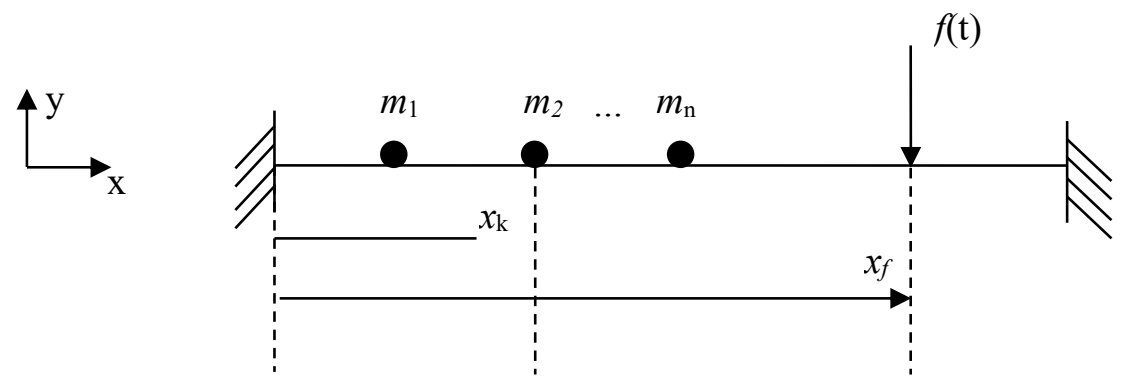

Fig. 1. A clamped-clamped beam with concentrated masses

Eq. (2) can be considered as the equation of forced vibration of a beam without concentrated masses which is acted by the inertia forces of $n$ concentrated masses and the external force $f(t)$. The solution of Eq. (2) can be expressed in the form

$$
y(x, t)=\sum_{i=1}^{\infty} \phi_{i}(x) q_{i}(t)
$$

where $\phi_{i}$ is the $i^{t h}$ mode shape of the beam without concentrated masses and $q_{i}$ is the $i^{t h}$ generalized coordinate.

Substituting (3) into (2), yields

$E I \sum_{i=1}^{\infty} \phi_{i}^{\prime \prime \prime \prime}(x) q_{i}(t)+m \sum_{i=1}^{\infty} \phi_{i}(x) \ddot{q}_{i}(t)=-\sum_{k=1}^{n} \delta\left(x-x_{k}\right) m_{k} \sum_{i=1}^{\infty} \phi_{i}(x) \ddot{q}_{i}(t)+\delta\left(x-x_{f}\right) f(t)$.

Multiplying Eq. (4) by $\phi_{j}(x)$ and integrating from 0 to $L$ and considering the definition of the Dirac delta function, one obtains

$$
\begin{aligned}
& \int_{0}^{L} E I \sum_{i=1}^{\infty} \phi_{i}{ }^{\prime \prime \prime \prime}(x) \phi_{j}(x) q_{i}(t) \mathrm{d} x+\int_{0}^{L} m \sum_{i=1}^{\infty} \phi_{i}(x) \phi_{j}(x) \ddot{q}_{i}(t) \mathrm{d} x \\
& =-\sum_{k=1}^{n} m_{k} \phi_{i}\left(x_{k}\right) \phi_{j}\left(x_{k}\right) \ddot{q}_{i}(t)+\phi_{j}\left(x_{f}\right) f(t) .
\end{aligned}
$$

The orthogonality of the normal mode shapes of the beam without concentrated masses can be addressed here

$$
\begin{array}{ll}
\int_{0}^{L} \phi_{i}(x) E I \phi_{i}^{\prime \prime \prime \prime}(x) \mathrm{d} x=0 & \text { if } i \neq j \\
\int_{0}^{L} \phi_{i}(x) m \phi_{j}(x) \mathrm{d} x=0 & \text { if } i \neq j
\end{array}
$$


Integrating the first equation in Eq. (6) twice by parts, yields

$$
\left.\phi_{i}(x) E I \phi_{j}^{\prime \prime \prime}(x)\right|_{0} ^{L}-\left.\phi_{i}^{\prime}(x) E I \phi_{j}^{\prime \prime}(x)\right|_{0} ^{L}+\int_{0}^{L} \phi_{i}^{\prime \prime}(x) E I \phi_{j}^{\prime \prime}{ }_{j}(x) \mathrm{d} x=0 \quad \text { if } i \neq j .
$$

For general boundary conditions the first two terms in Eq. (8) vanish. Thus, from Eq. (8) we have

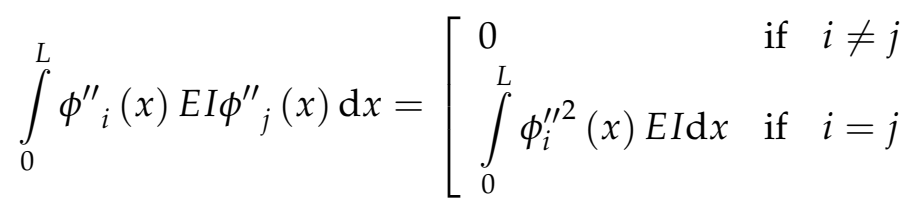

Applying Eqs. (6)-(9), Eq. (5) can be rewritten as

$$
\left[m \int_{0}^{L} \phi_{i}{ }^{2}(x) \mathrm{d} x+\sum_{k=1}^{n} m_{k} \phi_{i}^{2}\left(x_{k}\right)\right] \ddot{q}_{i}(t)+\left[E I \int_{0}^{L} \phi_{i}^{\prime \prime 2}(x) \mathrm{d} x\right] q_{i}(t)=\phi_{j}\left(x_{f}\right) f(t) \text {. }
$$

By introducing notations

$$
\begin{aligned}
& \mathbf{M}=m\left[\begin{array}{cccc}
\int_{0}^{L} \phi_{1}{ }^{2}(x) \mathrm{d} x+\sum_{k=1}^{n} \bar{m}_{k} \phi_{1}^{2}\left(x_{k}\right) & \sum_{k=1}^{n} \bar{m}_{k} \phi_{1}\left(x_{k}\right) \phi_{2}\left(x_{k}\right) & \ldots & \sum_{k=1}^{n} \bar{m}_{k} \phi_{1}\left(x_{k}\right) \phi_{N}\left(x_{k}\right) \\
\sum_{k=1}^{n} \bar{m}_{k} \phi_{2}\left(x_{k}\right) \phi_{1}\left(x_{k}\right) & \int_{0}^{L} \phi_{2}{ }^{2}(x) \mathrm{d} x+\sum_{k=1}^{n} \bar{m}_{k} \phi_{2}^{2}\left(x_{k}\right) & \ldots & \sum_{k=1}^{n} \bar{m}_{k} \phi_{2}\left(x_{k}\right) \phi_{N}\left(x_{k}\right) \\
\ldots & & & \ldots \\
\sum_{k=1}^{n} \bar{m}_{k} \phi_{N}\left(x_{k}\right) \phi_{1}\left(x_{k}\right) & \sum_{k=1}^{n} \bar{m}_{k} \phi_{N}\left(x_{k}\right) \phi_{2}\left(x_{k}\right) & \ldots & \int_{0}^{L} \phi_{N}^{2}(x) \mathrm{d} x+\sum_{k=1}^{n} \bar{m}_{k} \phi_{N}^{2}\left(x_{k}\right)
\end{array}\right], \\
& \mathbf{K}=E I\left[\begin{array}{cccc}
\int_{0}^{L} \phi^{\prime \prime 2}(x) \mathrm{d} x & 0 & \ldots & 0 \\
0 & \int_{0}^{L} \phi_{2}^{\prime \prime 2}(x) \mathrm{d} x & \ldots & 0 \\
\ldots & & & \ldots \\
0 & 0 & \ldots & \int_{0}^{L} \phi_{N}^{\prime \prime 2}(x) \mathrm{d} x
\end{array}\right], \\
& \boldsymbol{\Phi}(x)=\left[\phi_{1}(x), \ldots, \phi_{N}(x)\right]^{T}, \ddot{\mathbf{q}}(t)=\left[\ddot{q}_{1}(t), \ddot{q}_{2}(t), \ldots, \ddot{q}_{N}(t)\right]^{T} \text {, } \\
& \mathbf{q}(t)=\left[q_{1}(t), q_{2}(t), \ldots, q_{N}(t)\right]^{T}, \quad \bar{m}_{k}=\frac{m_{k}}{m} .
\end{aligned}
$$

Eq. (10) can be expressed in matrix form as follows

$$
\mathbf{M} \ddot{\mathbf{q}}(t)+\mathbf{K q}(t)=\boldsymbol{\Phi}\left(x_{f}\right) f(t) .
$$


The natural frequency of beam carrying concentrated masses can be obtained by solving the eigenvalue problem associated with Eq. (11), that is

$$
\operatorname{det}\left[\mathbf{K}-\omega^{2} \mathbf{M}\right]=0 \text {. }
$$

If the force is harmonic $f(t)=\bar{f} e^{i \omega t}$ then the solution of Eq. (11) can be found in the form

$$
\mathbf{q}(t)=\overline{\mathbf{q}} e^{i \omega t}
$$

Substituting Eq. (13) into Eq. (11) yields

$$
\left(\mathbf{K}-\omega^{2} \mathbf{M}\right) \overline{\mathbf{q}}=\boldsymbol{\Phi}\left(x_{f}\right) \bar{f} .
$$

The receptance function is defined as the frequency response function in which the response is the displacement. This means that in the frequency domain: receptance $=$ displacement/force. Thus, left multiplying Eq. (14) with $\frac{\boldsymbol{\Phi}^{T}(\xi)}{\bar{f}}\left[\left(\mathbf{K}-\omega^{2} \mathbf{M}\right)\right]^{-1}$ the receptance at $x$ due to the force at $x_{f}$ is obtained

$$
\alpha\left(x, x_{f}, \omega\right)=\frac{\boldsymbol{\Phi}^{T}(x) \overline{\mathbf{q}}}{\bar{f}}=\boldsymbol{\Phi}^{T}(x)\left(\mathbf{K}-\omega^{2} \mathbf{M}\right)^{-1} \boldsymbol{\Phi}\left(x_{f}\right) .
$$

It is noted that when infinite modes are applied, i.e. $N \rightarrow \infty$, Eq. (15) becomes the exact formula of the receptance function.

For the clamped-clamped beam, following relations can be derived:

$$
\begin{aligned}
& \phi_{i}(x)=\frac{\sin \alpha_{i} L+\sinh \alpha_{i} L}{\cos \alpha_{i} L-\cosh \alpha_{i} L}\left(\sin \alpha_{i} x-\sinh \alpha_{i} x\right)+\cos \alpha_{i} x-\cosh \alpha_{i} x, \\
& \phi^{\prime \prime}{ }_{i}(x)=-\alpha_{i}^{2}\left[\frac{\sin \alpha_{i} L+\sinh \alpha_{i} L}{\cos \alpha_{i} L-\cosh \alpha_{i} L}\left(\sin \alpha_{i} x+\sinh \alpha_{i} x\right)+\cos \alpha_{i} x+\cosh \alpha_{i} x\right], \\
& \int_{0}^{L} \phi_{i}^{2}(x) \mathrm{d} x=L, \\
& \int_{0}^{L}\left[\phi^{\prime \prime}{ }_{i}(x)\right]^{2} \mathrm{~d} x=L \alpha_{i}^{4},
\end{aligned}
$$

where $\alpha_{i}$ is the solution of the frequency equation $\cos \alpha L \cosh \alpha L-1=0$.

From Eq. (16) the matrices $\mathbf{M}$ and $\mathbf{K}$ are derived

$$
\mathbf{M}=m\left[\begin{array}{cccc}
L+\beta_{11} & \beta_{12} & \ldots & \beta_{1 N} \\
\beta_{21} & L+\beta_{22} & \ldots & \beta_{2 N} \\
\ldots & & & \ldots \\
\beta_{N 1} & \ldots & & L+\beta_{N N}
\end{array}\right], \quad \mathbf{K}=E I L\left[\begin{array}{cccc}
\alpha_{1}^{4} & 0 & \ldots & 0 \\
0 & \alpha_{2}^{4} & \ldots & 0 \\
\ldots & & \ldots \\
0 & \ldots & & \alpha_{N}^{4}
\end{array}\right] \text {, }
$$

where

$$
\begin{aligned}
\beta_{i j}=\sum_{k=1}^{n}\left\{\bar{m}_{k}\right. & {\left[\frac{\sin \alpha_{i} L+\sinh \alpha_{i} L}{\cos \alpha_{i} L-\cosh \alpha_{i} L}\left(\sin \alpha_{i} x_{k}-\sinh \alpha_{i} x_{k}\right)+\cos \alpha_{i} x_{k}-\cosh \alpha_{i} x_{k}\right] } \\
& \left.\times\left[\frac{\sin \alpha_{j} L+\sinh \alpha_{j} L}{\cos \alpha_{j} L-\cosh \alpha_{j} L}\left(\sin \alpha_{j} x_{k}-\sinh \alpha_{j} x_{k}\right)+\cos \alpha_{j} x_{k}-\cosh \alpha_{j} x_{k}\right]\right\} .
\end{aligned}
$$


The exact formula of the receptance of the clamped-clamped beam carrying concentrated masses will be derived from Eqs. (16)-(18).

\section{NUMERICAL SIMULATION}

\subsection{Reliability of the theory}

In order to check the reliability of the proposed receptance, frequency parameters $\alpha_{i} L$ of a clamped-clamped beam carrying two masses are calculated from Eq. (12) and compared to Ref. [14]. Five lowest frequency parameters of the clamped-clamped beam with two concentrated masses $\bar{m}_{1}=\bar{m}_{2}=0.5$ attached at $0.25 \mathrm{~L}$ and $0.75 \mathrm{~L}$ obtained by two methods are listed in Tab. 1. As can be seen from this table, the first five frequency parameters of the present work are in excellent agreement with Ref. [14]. This result justifies the reliability of the proposed receptance function.

Table 1. Frequency parameters of the clamped-clamped beam

\begin{tabular}{crcc}
\hline Frequency parameters & Ref. [14] & Present paper & Error $(\%)$ \\
\hline$\alpha_{1} L$ & 4.0973 & 4.0976 & 0.00007 \\
$\alpha_{2} L$ & 5.8984 & 5.8995 & 0.00019 \\
$\alpha_{3} L$ & 9.1453 & 9.1534 & 0.00089 \\
$\alpha_{4} L$ & 13.7527 & 13.7567 & 0.00029 \\
$\alpha_{5} L$ & 16.9258 & 16.9399 & 0.00083 \\
\hline
\end{tabular}

\subsection{Influence of location of the concentrated masses on the receptance}

In this paper, the numerical simulations of a clamped-clamped beam with two masses are presented. Parameters of the beam are: Mass density $\rho=7800 \mathrm{~kg} / \mathrm{m}^{3}$; modulus of elasticity $E=2.0 \times 1011 \mathrm{~N} / \mathrm{m}^{2} ; L=1 \mathrm{~m} ; b=0.02 \mathrm{~m} ; h=0.01 \mathrm{~m}$. Two equal concentrated masses of $0.6 \mathrm{~kg}$ are attached on the beam in different scenarios. The first five mode shapes are used to calculate the receptance. The receptance matrices are calculated at 50 points spaced equally on the beam while the force moves along these points.

The receptance of the clamped-clamped beam without masses is calculated first. Fig. 2 presents the receptance matrices when the forcing frequencies equal to the first, second and third natural frequencies of the beam-mass system, respectively. As can be seen from Fig. 2(a) when the forcing frequency is equal to the first natural frequency, the receptance is maximum at the middle of the beam which corresponds to the position where the amplitude of the first mode is maximum. As can be observed from Fig. 2(b) that when the forcing frequency is equal to the second natural frequency, the receptance is maximum at position of about $0.3 \mathrm{~L}$ and $0.7 \mathrm{~L}$ from the left end of the beam which are the positions where the amplitude of the second mode shape is maximum. Meanwhile, the receptance is smallest at the middle of beam which corresponds to the position where the amplitude of the second mode shape is minimum. Fig. 2(c) presents the receptance matrix of the beam when the frequency of the force is equal to the third natural frequency. The receptance of the beam is maximum at the positions of about $0.2 \mathrm{~L}, 0.5 \mathrm{~L}$ and 
$0.8 \mathrm{~L}$ where the amplitude of the third mode shape is maximum. The receptance is minimum at positions of about $0.35 \mathrm{~L}, 0.65 \mathrm{~L}$ where the amplitude of the third mode shape is minimum. It can be concluded that, when the excitation frequency is equal to a natural frequency the positions of maxima and minima in the receptance are the same with the positions of maxima and minima in the corresponding mode shape. Therefore, similar to the mode shape, we call the maxima in the receptance "peaks of receptance" and the minima in the receptance "nodes of receptance".

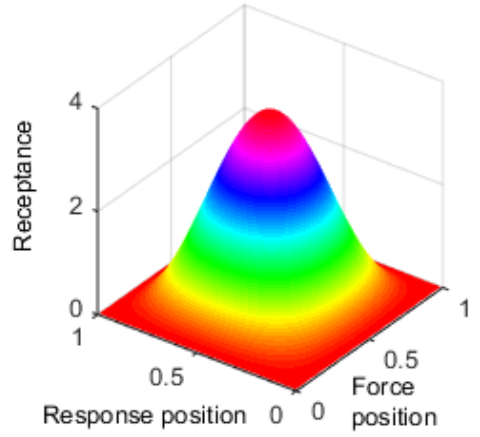

(a) $\omega=\omega_{1}$

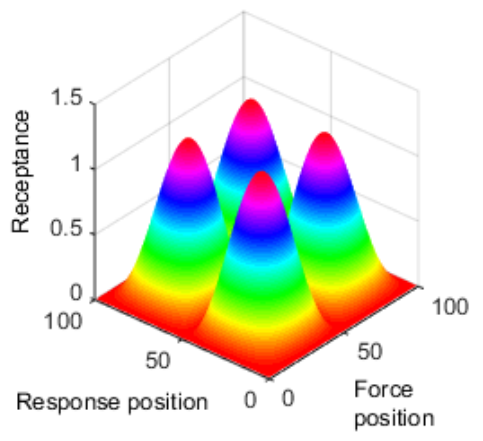

(b) $\omega=\omega_{2}$

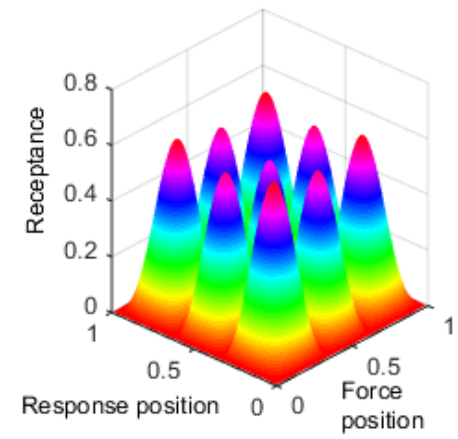

(c) $\omega=\omega_{3}$

Fig. 2. Receptance of beam without a masse

When there is a concentrated mass, the receptance matrix of the beam is changed. Fig. 3 presents the receptance matrices of the beam when the forcing frequency is equal to the first natural frequency of the beam-mass system. As can be seen from this figure, when the mass is located $0.25 \mathrm{~L}$ the position of the peak of receptance "moves" to the left end of beam. However, when the mass is located at the middle of the beam, the shape of the receptance is unchanged. The change of position of the peak of receptance is depicted clearer in Fig. 4 when the force is fixed at position $0.5 \mathrm{~L}$. As can be observed from this figure, the peak of receptance moves to the position of $0.4 \mathrm{~L}$ when the mass is located at $0.25 \mathrm{~L}$. The receptance seems to be "pulled" toward the mass position.

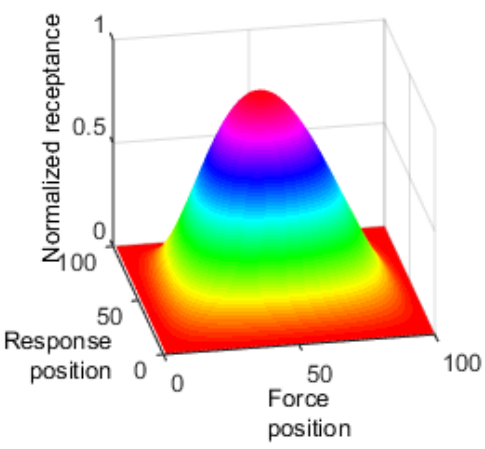

(a) Mass is at $0.25 \mathrm{~L}$

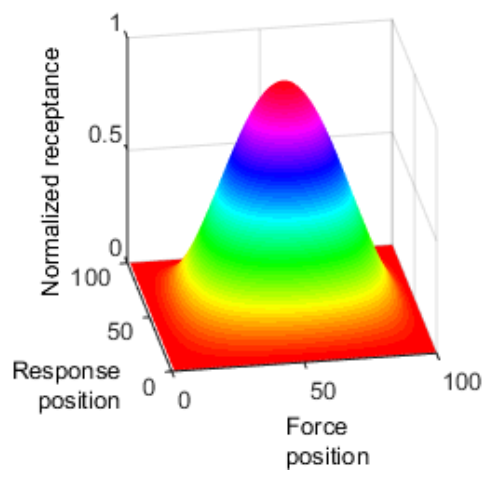

(b) Mass is at $0.5 \mathrm{~L}$

Fig. 3. Receptance matrices at $\omega=\omega_{1}$ 


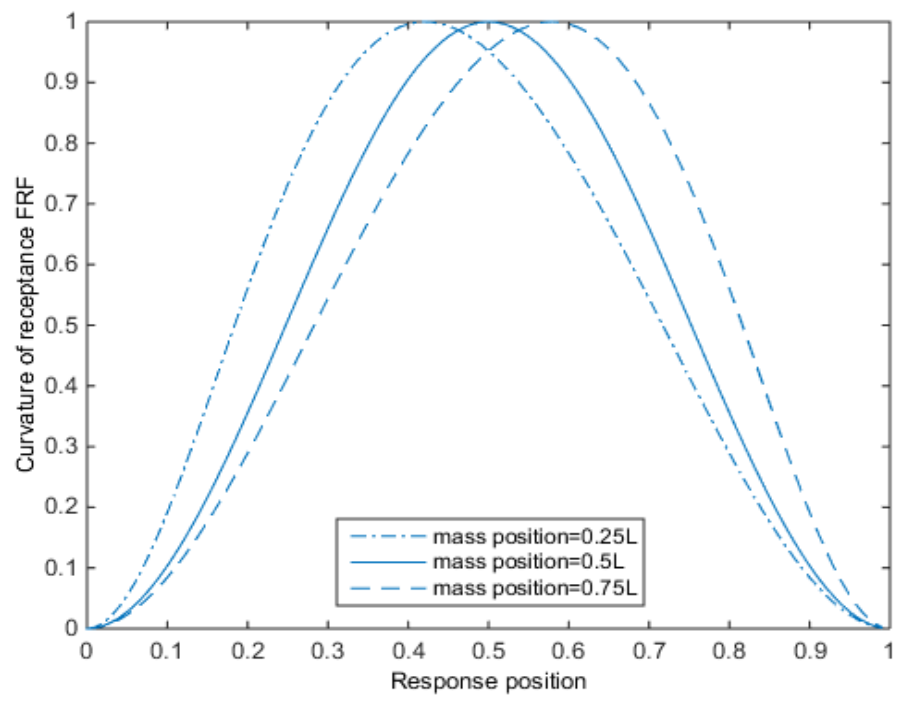

Fig. 4. Receptance of beam with force position is at $L / 2, \omega=\omega_{1}$

Fig. 5 presents the receptance of beam carrying a concentrated mass at different positions when the forcing frequency is equal to the second natural frequency. As shown in Fig. 5(a), when the mass is located at $0.3 \mathrm{~L}$, the peaks corresponding to either the response position of $0.3 \mathrm{~L}$ or the force position of $0.3 \mathrm{~L}$ decrease significantly. When the mass is located the middle of the beam, the receptance shape is unchanged as shown in Fig. 5(b). These results show that when the mass is attached at a peak of the receptance matrix, the peaks corresponding to either the response position or force position which is close to the mass position will decrease. Meanwhile, the shape of receptance is unchanged when the mass is attached at the nodes of the receptance. The change in receptance can be observed in more detail as presented in Fig. 6 when the force is fixed at position $0.25 \mathrm{~L}$. As can be seen from this figure, the peak of receptance decreases. In addition, the peak of receptance moves slightly toward the mass position.

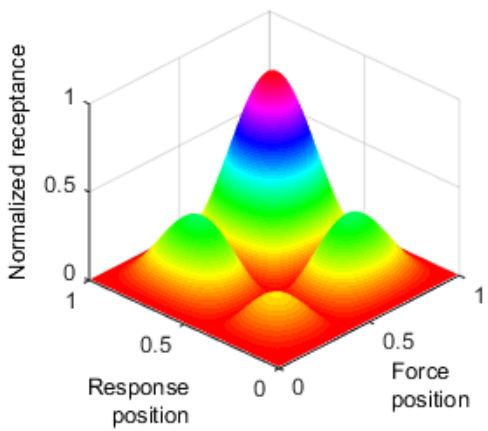

(a) Mass is at $0.25 \mathrm{~L}$

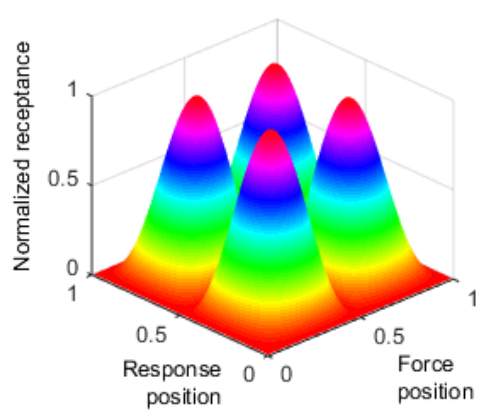

(b) Mass is at $0.5 L$

Fig. 5. Receptance of beam at $\omega=\omega_{2}$ 
Theoretical and experimental analysis of the exact receptance function of a clamped-clamped beam with concentrated masses 37

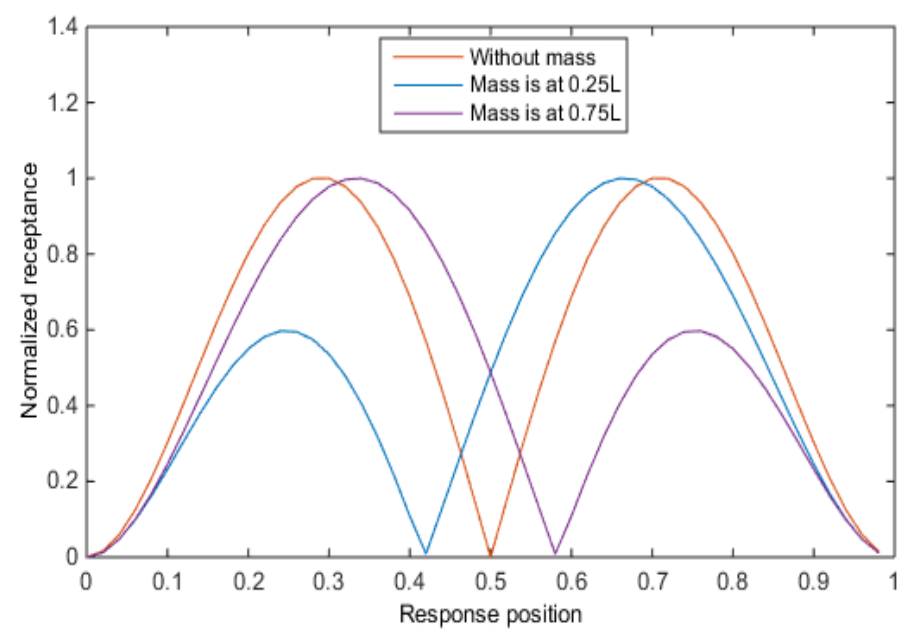

Fig. 6. Measured receptance with the force acting at $0.25 L, \omega=\omega_{2}$

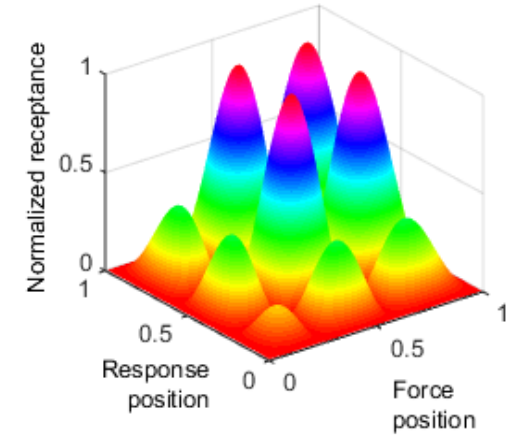

(a) Mass is at $0.2 L$

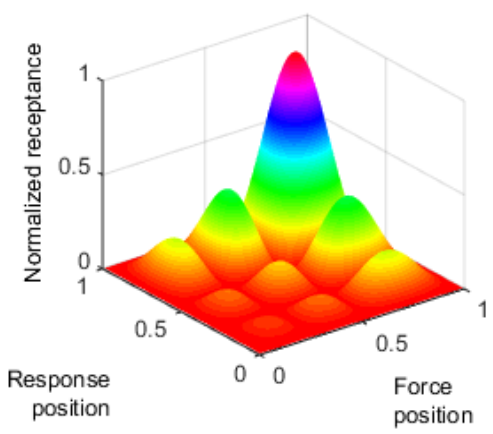

(c) Masses are at $0.2 \mathrm{~L}$ and $0.5 \mathrm{~L}$

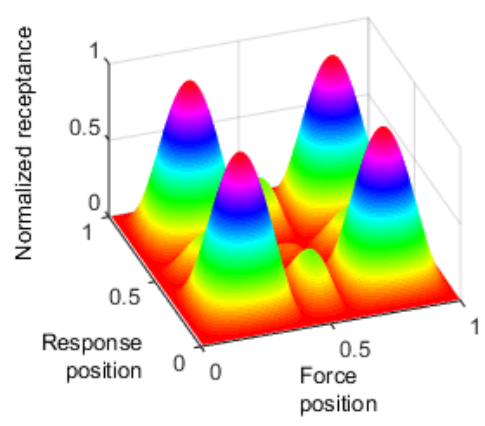

(b) Mass is at $0.5 \mathrm{~L}$

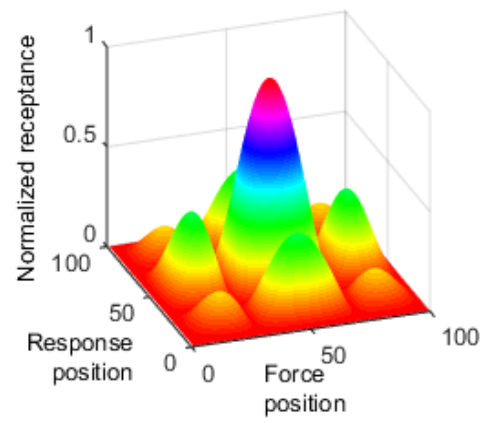

(d) Masses are at $0.2 \mathrm{~L}$ and $0.8 \mathrm{~L}$

Fig. 7. Normalized receptance at $\omega=\omega_{3}$

Fig. 7 presents the receptance of beam when the forcing frequency is equal to the third natural frequency. As shown in Fig. 7(a), when one mass is located at $0.2 \mathrm{~L}$ or $0.8 \mathrm{~L}$, 
the peaks corresponding to either the response position of $0.2 L$ or the force positions of $0.2 \mathrm{~L}$ decrease significantly. When one mass is located at $0.5 \mathrm{~L}$, the peaks corresponding to either the response position of $0.5 \mathrm{~L}$ or the force position of $0.5 \mathrm{~L}$ decrease significantly as shown in Fig. 7(b). When two masses are located at $0.2 \mathrm{~L}$ and $0.5 \mathrm{~L}$, the peaks corresponding to either the response positions of $0.2 \mathrm{~L}, 0.5 \mathrm{~L}$ or the force positions of $0.2 \mathrm{~L}, 0.5 \mathrm{~L}$ decrease significantly as depicted in Fig. 7(c). When two masses are located at $0.2 \mathrm{~L}$ and $0.8 \mathrm{~L}$, the peaks corresponding to either the response positions of $0.2 L, 0.8 \mathrm{~L}$ or the force positions of $0.2 L, 0.8 L$ decrease significantly as shown in Fig. $7(\mathrm{~d})$. The change in receptance can be seen in more detail when the force is fixed at position $0.2 \mathrm{~L}$ as depicted in Fig. 8. Similar conclusion can be drawn from this figure that when the masses attached at peaks, these peaks will decrease significantly. When there is one mass the peaks of receptance move toward the mass position. When there are two masses attached symmetrically at $0.2 \mathrm{~L}$ and $0.8 \mathrm{~L}$ the peak at $0.2 \mathrm{~L}$ moves to the left end, while the peak at $0.8 \mathrm{~L}$ moves to the right end. When there are two masses attached at $0.2 \mathrm{~L}$ and $0.5 \mathrm{~L}$, the receptance is "pulled" to the left end. In this case, the receptance tends to "move" toward the heavier side of the beam.

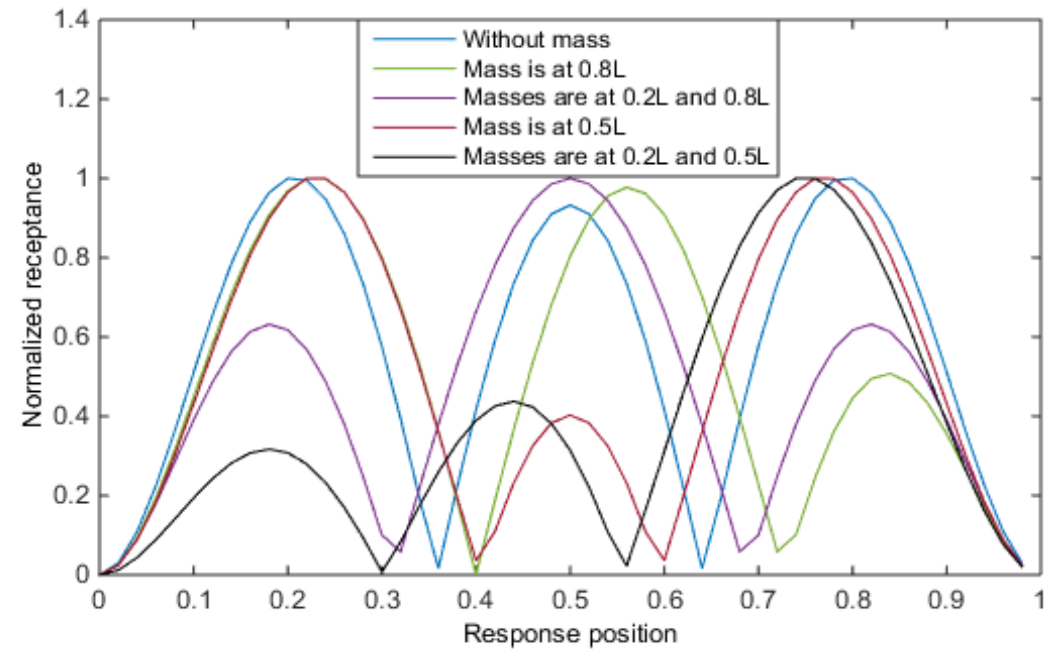

Fig. 8. Normalized receptance when the force is fixed at $0.2 L, \omega=\omega_{3}$

\section{EXPERIMENT RESULTS}

The experimental setup is illustrated in Fig. 9. The clamped-clamped beam with the same parameters presented in Section 3.1 has been tested. The beam is excited by the Vibration Exciter Bruel \& Kjaer 4808 and the response is measured by the instrument Polytec Laser Vibrometer PVD-100. Two equal concentrated masses of $0.6 \mathrm{~kg}$ are attached on the beam in different scenarios. The receptance is measured along the beam when the forcing frequency is set to the first three natural frequencies of the beam-mass system. The receptance matrix is obtained at 50 points spaced equally on the beam.

According to the simulation results, when the forcing frequency is equal to the first natural frequency, the change in receptance is simple that it has only one peak at the middle of the beam and it moves toward the position of the attached mass. Meanwhile, when 


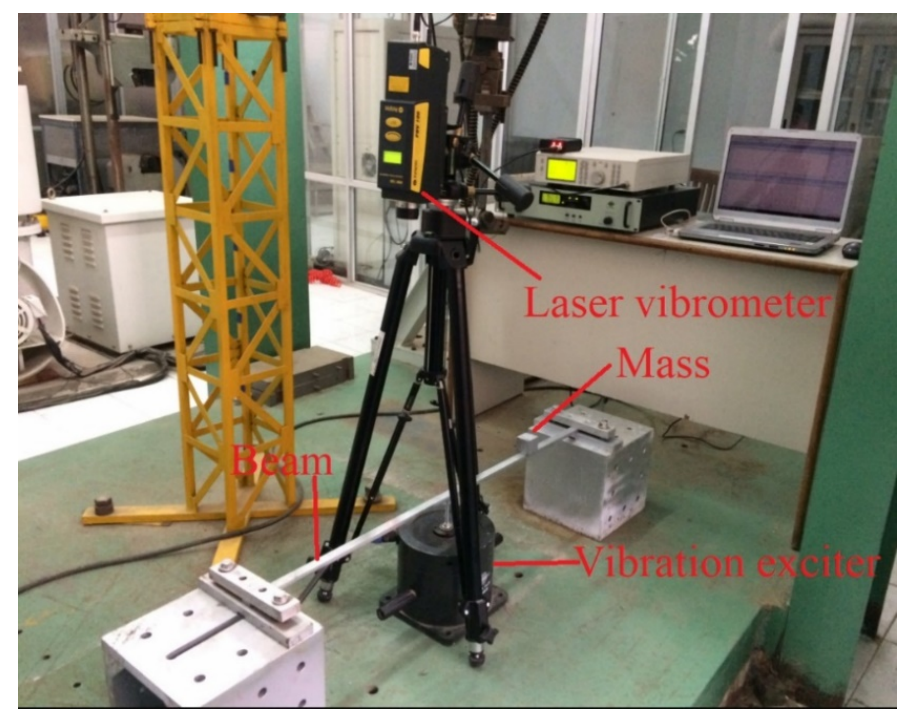

Fig. 9. Experimental setup

the forcing frequency is high the change in receptance becomes more complicated with different configurations of the attached masses. Therefore, when the forcing frequency is equal to the first natural frequency only the receptance curve extracted with the force fixed at one position is measured, while the whole receptance matrices are measured at the second and third natural frequencies.

When the mass is attached at the position of $L / 4$, the force is fixed at $L / 2$ and the forcing frequency is equal to the first natural frequency, the measured receptance moves to the left end as presented in Fig. 10. Comparing Figs. 4 and 10 it is concluded that the measured receptance and the simulation results are in very good agreement in both cases without and with an attached mass.

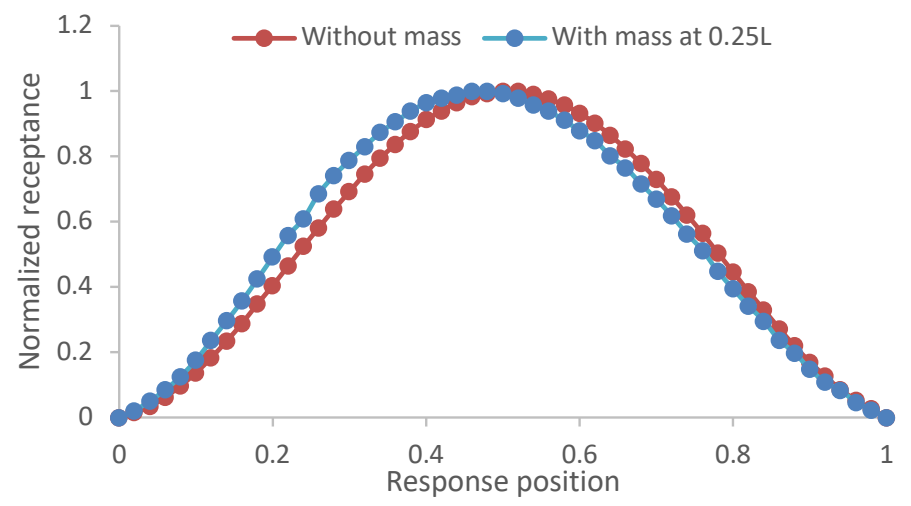

Fig. 10. Measured receptance curves of beam, force position $=L / 4, \omega=\omega_{1}$ 
When the excitation frequency is equal to the second natural frequency and the mas is attached at the position of $L / 4$, the measured receptance matrix presented in Fig. 5(a) and the simulation receptance matrix shown in Fig. 11(a) are in very good agreement. As can be seen from Fig. 11(a), three peaks corresponding to the position of $L / 4$ in the receptance matrix decrease significantly. When the mass is attached at $L / 2$ and the forcing frequency is equal to the third natural frequency, five peaks of the receptance matrix corresponding to the position of $L / 2$ decrease significantly as can be observed in Fig. 11(b). This agrees with the simulation result depicted in Fig. 7(b). Fig. 12 presents the experimental receptance curves of beam without and with an attached mass at $L / 4$ which was measured when the forcing frequency is equal to the second natural frequency. When there is no mass attached, these receptance has two peaks at $L / 4$ and $3 L / 4$. These experimental results justify the correctness of the simulation results presented in Fig. 6 . When there is a mass attached at the position of $L / 4$, the peak at the mass position decreases clearly. Fig. 13 presents the receptance measured when the force frequency is equal to the third natural frequency. As can be seen from this figure, when there is no mass attached the receptance has three peaks at $L / 6, L / 2$ and $5 L / 6$. When there are masses attached,

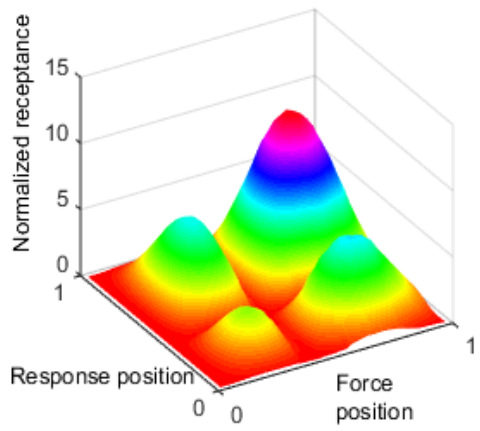

(a) $\omega=\omega_{2}$

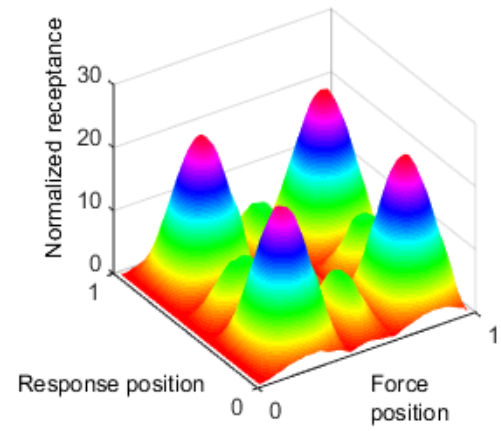

(b) $\omega=\omega_{3}$

Fig. 11. Measured receptance matrices of beam

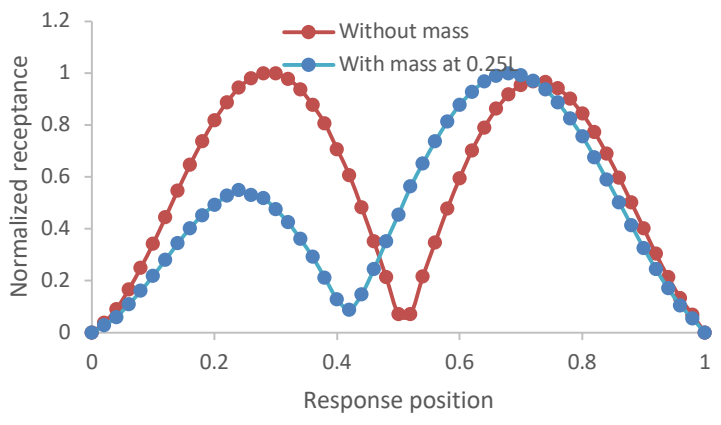

Fig. 12. Measured receptance curves of beam, force position $=L / 4, \omega=\omega_{2}$

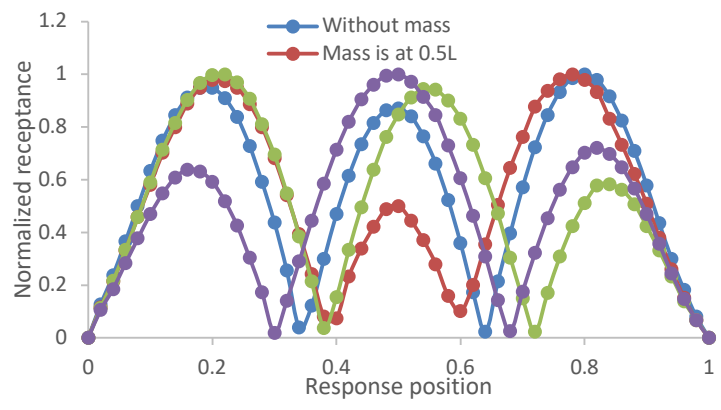

Fig. 13. Measured receptance curves of beam, force position $=L / 6, \omega=\omega_{3}$ 
the receptance peaks decrease significantly at the mass positions. The experimental results presented in Fig. 13 are in very good agreement with the simulation results depicted in Fig. 8.

\section{CONCLUSIONS}

In this paper, the exact receptance function of clamped-clamped beam carrying concentrated masses is derived. The proposed receptance function can be applied easily for predicting the response of the beam under a harmonic excitation. The influence of the concentrated masses on the receptance of beam is also investigated. When the excitation frequency is equal to a natural frequency, the peaks and nodes positions of the receptance are the same with the maximum and minimum positions of the corresponding mode shape. When there are concentrated masses the shape of receptance is changed. When the mass positions are close to peaks of receptance, these peaks will decrease significantly. When the masses are located at the nodes of receptance, the receptance is not influenced. The influence of masses on the receptance matrices can be used to control the vibration amplitudes at some specific positions at given forcing frequencies.

The experiment has been carried out when the forcing frequency is set to the first three natural frequencies of the beam carrying concentrated masses. The experimental and simulation results are in very good agreement which justifies the proposed method.

\section{ACKNOWLEDGEMENTS}

This research is funded by Vietnam National Foundation for Science and Technology Development (NAFOSTED) under grant number 107.02-2017.300.

\section{REFERENCES}

[1] R. E. D. Bishop and D. C. Johnson. The mechanics of vibration. Cambridge University Press, (2011).

[2] H. K. Milne. The receptance functions of uniform beams. Journal of Sound and Vibration, 131, (3), (1989), pp. 353-365. https://doi.org/10.1016/0022-460X(89)90998-X.

[3] B. Yang. Exact receptances of nonproportionally damped dynamic systems. Journal of Vibration and Acoustics, 115, (1), (1993), pp. 47-52. https://doi.org/10.1115/1.2930313.

[4] R. M. Lin and M. K. Lim. Derivation of structural design sensitivities from vibration test data. Journal of Sound and Vibration, 201, (5), (1997), pp. 613-631. https://doi.org/10.1006/jsvi.1996.0836.

[5] J. E. Mottershead. On the zeros of structural frequency response functions and their sensitivities. Mechanical Systems and Signal Processing, 12, (5), (1998), pp. 591-597. https://doi.org/10.1006/mssp.1998.0167.

[6] M. Gurgoze. Receptance matrices of viscously damped systems subject to several constraint equations. Journal of Sound and Vibration, 230, (5), (2000), pp. 1185-1190. https://doi.org/10.1006/jsvi.1999.2602.

[7] M. Gürgöze and H. Erol. On the frequency response function of a damped cantilever simply supported in-span and carrying a tip mass. Journal of Sound and Vibration, 255, (3), (2002), pp. 489-500. https://doi.org/10.1006/jsvi.2001.4118. 
[8] A. Burlon, G. Failla, and F. Arena. Exact frequency response analysis of axially loaded beams with viscoelastic dampers. International Journal of Mechanical Sciences, 115, (2016), pp. 370384. https://doi.org/10.1016/j.ijmecsci.2016.07.024.

[9] A. Burlon, G. Failla, and F. Arena. Exact frequency response of two-node coupled bendingtorsional beam element with attachments. Applied Mathematical Modelling, 63, (2018), pp. 508537. https://doi.org/10.1016/j.apm.2018.06.047.

[10] A. Karakas and M. Gürgöze. A novel formulation of the receptance matrix of nonproportionally damped dynamic systems. Journal of Sound and Vibration, 264, (3), (2003), pp. 733-740. https://doi.org/10.1016/s0022-460x(02)01507-9.

[11] G. Muscolino, R. Santoro, and A. Sofi. Explicit frequency response functions of discretized structures with uncertain parameters. Computers \& Structures, 133, (2014), pp. 64-78. https://doi.org/10.1016/j.compstruc.2013.11.007.

[12] N. V. Khoa, C. Van Mai, and D. T. B. Thao. Exact receptance function and receptance curvature of a clamped-clamped continuous cracked beam. Vietnam Journal of Mechanics, 41, (4), (2019), pp. 349-361. https://doi.org/10.15625/0866-7136/14566.

[13] J. S. Wu and T. L. Lin. Free vibration analysis of a uniform cantilever beam with point masses by an analytical-and-numerical-combined method. Journal of Sound and Vibration, 136, (2), (1990), pp. 201-213. https://doi.org/10.1016/0022-460X(90)90851-P.

[14] S. Maiz, D. V. Bambill, C. A. Rossit, and P. A. A. Laura. Transverse vibration of Bernoulli-Euler beams carrying point masses and taking into account their rotatory inertia: Exact solution. Journal of Sound and Vibration, 303, (3-5), (2007), pp. 895-908. https://doi.org/10.1016/j.jsv.2006.12.028. 